\title{
AdjusTable 3D Plasmonic Archimedes Spiral Lens for Optical Manipulation
}

\author{
Lin Cheng *, Xiaomingliang Li, Zelong Wang, Pengfei Cao, Xiaodong He and Tiaoming Niu \\ School of Information Science and Engineering, Lanzhou University, Lanzhou 730000, China; \\ lixml16@lzu.edu.cn (X.L.); wangzl17@lzu.edu.cn (Z.W.); caopf@lzu.edu.cn (P.C.); xdhe@lzu.edu.cn (X.H.); \\ niutm@lzu.edu.cn (T.N.); \\ * Correspondence: chenglin@lzu.edu.cn;
}

Received: 7 January 2019; Accepted: 11 February 2019; Published: 16 February 2019

\begin{abstract}
A novel adjustable three-dimensional plasmonic Archimedes spiral lens (3D PASL) has been investigated and analyzed in detail by numerical simulations. The 3D PASL consists of a spiraling Archimedes helix slot that is engraved on the inner wall of a funnel-shaped gold film on a silicon dioxide substrate. When the incident light from the bottom of substrate is composed of left-hand circularly polarized (LCP) waves, the transmitted light field will converge completely to a focused point that floats in the hollow funnel. This light field will change into an optical vortex when the incident light is changed to right-hand circularly polarized (RCP) waves. The performance of our 3D PASL is discussed for particle trapping or rotation applications. In addition, the position of the optical focus or vortex can be adjusted by varying the height of the structure. Our 3D PASL is highly flexible for practical optical manipulation applications and overcomes the problem where the previous two-dimensional PASL could only manipulate particles on the surface.
\end{abstract}

Keywords: plasmonic; archimedes spiral; optical manipulation

\section{Introduction}

Surface plasmons (SPs) are waves that result from the collective oscillations of electrons excited by electromagnetic waves at a metal/dielectric interface, propagating along the metal surface and evanescently confined in the perpendicular direction [1-6]. SP waves have local confinement characteristics, and their distribution depths are smaller than the wavelength order, i.e., beyond the diffraction limit [2,7], making SPs a great candidate for use in the production of sub-wavelength optoelectronic devices [8-12]. By controlling the propagation of SPs at the nanoscale, functions such as focusing and trapping nanoparticles at a much lower intensity level can be achieved [13,14]. In conventional optics, however, this is not possible, because of the limitations of the diffraction limit, and the trapping force drops quickly as the radius of the particle decreases due to reduced gradient force [15], so the excellent performance of this beyond-the-diffraction-limit technology is used fully to design SP lenses [16-18] and plasmonic trapping devices [19]. The Archimedes spiral lens gives incident light an additional geometric phase for different handedness [20-23], and the light field will thus exhibit different functions for particle trapping or particle rotation [14,24,25]. In contrast to the traditional 2D plasmonic Archimedes spiral lens (PASL), the Archimedes spirals were engraved on a planar gold film, and a focusing spot or plasmonic vortex can only be formed by constructive interference on the gold film surface when the distance between the two spirals is equal to the surface plasmon polariton (SPP) wavelength. This greatly reduces flexibility for practical applications of these PASLs, such as manipulation of biological cells [26] and stretching of DNA $[27,28]$.

In this work, a single three-dimensional (3D) PASL has been investigated by finite element method (FEM). By controlling the angular momentum of the plasmonic near-field directly, a focusing 
spot or a plasmonic vortex field can be formed in the central hollow of the 3D PASL. We study the effects of several parameters, such as the initial spiral radius $r_{0}$, the horizontal distance between successive turns of the spiral gap and the height of the structure $h_{0}$, on the focusing spot/plasmonic vortex position and the focusing intensity in detail. We find that the focusing spot/plasmonic vortex position can only be adjusted by varying the height of the structure. It should be noted that our 3D PASL is free from the rigid structural constraint of the adjacent helical spiral pitch that is included in the 2D PASL for the sake of constructive interference. This makes our designed 3D PASL structure much more flexible, and it offers greater potential than the 2D PASL for many practical applications, including micromechanical systems, optofluidic devices, microbiology and biomedical photonics, particularly in the analysis of conformational changes in DNA or proteins by providing controllable trapping or a controllable local vortex turbulence [29].

\section{Plasmonic Archimedes Spiral Lens}

Normally, the geometry of an Archimedes spiral (AS) is expressed using polar coordinates as $r(\phi)=r_{0}+(\phi * g a p) / 2 \pi$, where $r$ is the distance from the AS to the origin $\mathrm{O}, r_{0}$ is the initial spiral radius, $\phi$ is the azimuthal angle in radians, and gap represents the horizontal distance between successive turns of the spiral. Here, we extrude the two-dimensional Archimedean spiral into three dimensions along the $z$-axis using the relation $z=h_{0} * \phi / 2 \pi$, where $h_{0}$ is the rise height of the 3D AS when $\phi$ grows by $2 \pi$. Obviously, if $h_{0}=0$, then the 3D structure reverts to its $2 \mathrm{D}$ counterpart.

In this manuscript, we design a 3D PASL, as shown in Figure 1. The 3D PASL is fabricated on a silicon dioxide substrate that has a funnel-shaped depression, which is plated with a 300-nm-thick gold film. A 3D Archimedes spiral slot is then engraved by swirling on the inner sidewall of the funnel-shaped gold film. Please note that both the bottom of the silicon dioxide substrate and gold film are closed to avoid the directly transmitted light interfering with the plasmonic field. The ambient medium in the funnel and above the gold film is buffer solution (such as phosphate buffer saline, abbreviated PBS, a water-based pH-adjusted blend of ultrapure-grade phosphate buffers and saline solutions. The contents of PBS buffer are determined by the requirements of concentration of salt in the buffer and $\mathrm{pH}$ in practical application. The $\mathrm{pH}$ of most common composition of PBS is 7.4.), which is feasible to represent the real cell trapping environment. The slot is approximately $200 \mathrm{~nm}$ wide and starts at $r_{0}=1.4 \mu \mathrm{m}$, with its azimuthal angle $\phi$ ranging from 0 to $6 \pi$, i.e., the completed helix has three turns.

When the circularly polarized light propagating along the $z$-axis illuminates the 3D PASL from the bottom of the $\mathrm{SiO}_{2}$ substrate, the incident light wave is coupled to the SPP modes at the entrances to the AS slot. SPPs, as evanescent waves, propagate along the radial direction, either inwards or outwards, to the center of the PASL. Because of the decoupling effect of the 3D spiral slots, some of the SPPs are scattered into free space and are then converted into propagating waves when they encounter other out-of-plane spiral grooves. This is the main point by which our 3D polycyclic AS structure is quite different from its 2D counterpart. These propagating waves interfere and produce high-intensity focal spots or plasmonic vortices at the center of the structure, as shown in Figure 1c,d, which will be discussed in the next section.

In this paper, the optical excitation of this PASL involves the use of left-hand or right-hand circularly polarized plane waves, which carry spin angular momentum $s=1$ or $s=-1$, respectively. Because of the complexity of the three-dimensional structure, we changed the traditional two-dimensional Archimedes spiral electric field distribution equation [30] and we only study the electric field distribution on the central axis of the funnel. When illuminated by light with left-hand circular polarization, the SP field generated along the $z$-direction on the central axis of the structure can be expressed in cylindrical coordinates as:

$$
E_{Z}(h)=E_{0} \int e^{i\left(\phi+\frac{\phi \cdot h_{0}}{\lambda_{d}}\right)} e^{-i k_{s p p} \cdot \cos \theta \cdot r_{d}} \sqrt{r^{2}(\phi)+\frac{g a p^{2}+h_{0}^{2}}{4 \pi^{2}}} d \phi
$$


Here, $h$ represents the height of a specified point A on the central axis of the structure, $k_{s p p}$ is the wavenumber of the generated SPP, $r_{d}$ is the distance from a point on the spiral slot to the point A on the central axis of the structure, and $\lambda_{d}$ is the wavelength of the incident light in the $\mathrm{SiO}_{2} \cdot k_{s p p} \cdot \cos \theta$ is a component of the SPP wave vector and $\phi h_{0} / \lambda_{d}$ is the additional phase caused by the increase in the spiral's height. Using the above equation, we can obtain the relationship between $h_{0}$ and the focal length, and we find that the focal length also increases when $h_{0}$ increases within a specific range.

To enable convergence and focusing of a scattering field using the polycyclic AS structure, the relationship between the structural parameters and the additive phase difference must satisfy the following equation: $\Delta r=\Delta \phi \lambda_{s p p} / 2 \pi$, where $\Delta r$ is the increment in distance between two points on the spiral slot relative to the focal point, and $\Delta \phi$ is the additive phase difference between these two points on the spiral. The phase difference consists of two parts; one is the geometric phase that is caused by the AS itself, and the other is simply caused by the rise in height of the 3D spiral that is equal to $\Delta h \cdot 2 \pi / \lambda_{d}$, where $\Delta h$ is the height increment between the two points on the spiral. Obviously, when $\Delta \phi \lambda_{s p p} / 2 \pi$ is equal to the difference in distance between the spiral slot and a point at the center of the structure, a focal point will be generated.

(a)

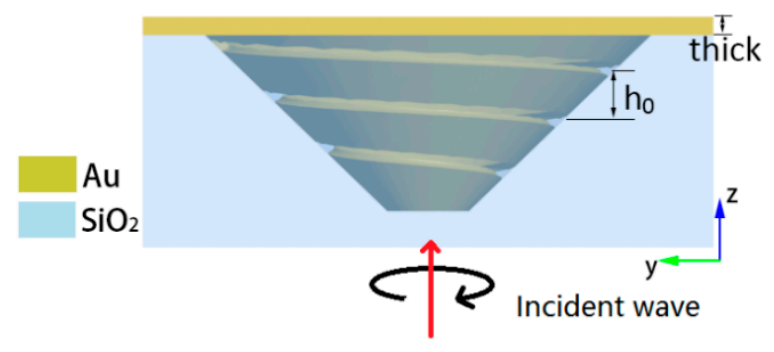

(b)

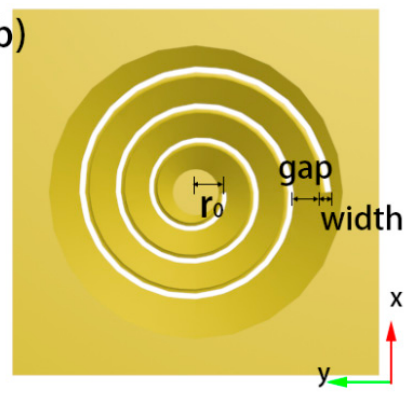

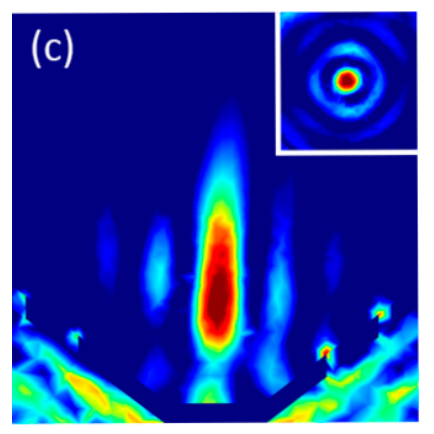

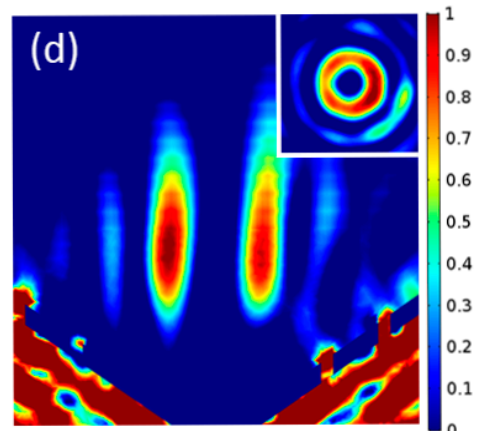

Figure 1. Schematics of the geometry of a 3D plasmonic Archimedes spiral lens from (a) the side view and (b) the top view. Cross-sectional intensity distributions of the 3D PASL under normally incident excitation with (c) left-hand and (d) right-hand circular polarization, where the insets on the upper right represent the corresponding intensity distributions from the top view. FEM simulation parameters: illumination wavelength $\lambda=1310 \mathrm{~nm}, r_{0}=1400 \mathrm{~nm}$, gap $=800 \mathrm{~nm}$, thickness $=300 \mathrm{~nm}$, width $=200 \mathrm{~nm}$, and $h_{0}=625 \mathrm{~nm}$.

\section{Simulations and Discussion}

To verify the performance of the proposed 3D PASL, finite element method (FEM) simulations are performed using Comsol Multiphysics 5.2a software. In our simulation, the power of the incident light is $80 \mathrm{mw}$, the free space wavelength of the incident light is $1310 \mathrm{~nm}$ and the dielectric constant of gold at this wavelength is $\varepsilon_{m}=-68.53+7.4542 \mathrm{i}$ [31]. Consequently, the wavelength of the SPP that is launched at the Au-PBS interface is $\lambda_{s p p}=969 \mathrm{~nm}$, which is calculated from $\lambda_{s p p}=\lambda_{0} \sqrt{\left(\varepsilon_{m}^{\mathrm{Re}}+\varepsilon_{d}\right) /\left(\varepsilon_{m}^{\mathrm{Re}} \varepsilon_{d}\right)}$, where $\varepsilon_{m}^{\mathrm{Re}}$ represents the real part of $\varepsilon_{m}$ and the dielectric constant of the ambient PBS buffer solution is $\varepsilon_{d}=1.7408$. 
The above equations for the 3D AS indicate that the main factor that affects the focal length of the proposed 3D structure is the rise height $h_{0}$ of the 3D PASL, which can be expected to influence the generated SP near-field. Therefore, we focus here on discussing the effects of this factor on the focal length under excitation by light with left-hand circular polarization.

Through numerical analysis, we found that, compared with parameters such as gap and $r_{0}, h_{0}$ has the most obvious effect on the focal length. We therefore fixed the parameters gap, $r_{0}$ and width at $800 \mathrm{~nm}, 1400 \mathrm{~nm}$ and $200 \mathrm{~nm}$, respectively, and then gradually changed the rise height $h_{0}$ between successive turns of the spiral from $575 \mathrm{~nm}$ to $725 \mathrm{~nm}$ in steps of $50 \mathrm{~nm}$. Figure 2a-d shows the intensity distributions $\left(|\mathrm{E}|^{2}\right)$ for different $h_{0}$ values under illumination by incident light with left-hand circular polarization. It was found that the focus position floats above the funnel shape and moves further from the bottom of the funnel with increasing $h_{0}$. Because the 3D spiral groove rises simultaneously, this also causes the focus to rise. Unlike its counterpart 2D structure composed of three concentric annular rings, a slight asymmetry in the intensity is expected to be observed when the damping of the SPP in both media and the unequal propagation distances from the spiral slot to the focus over the different azimuthal angles are taken into account. The focusing characteristics of the 3D PASL are depicted in Figure 3. The figure shows that the focal length is elongated by $656 \mathrm{~nm}$ (from $2765 \mathrm{~nm}$ to $3421 \mathrm{~nm}$ ) when $h_{0}$ increases by $150 \mathrm{~nm}$. Figure $3 \mathrm{~b}$ indicates that there is no obvious change in the value of the full width at half maximum.
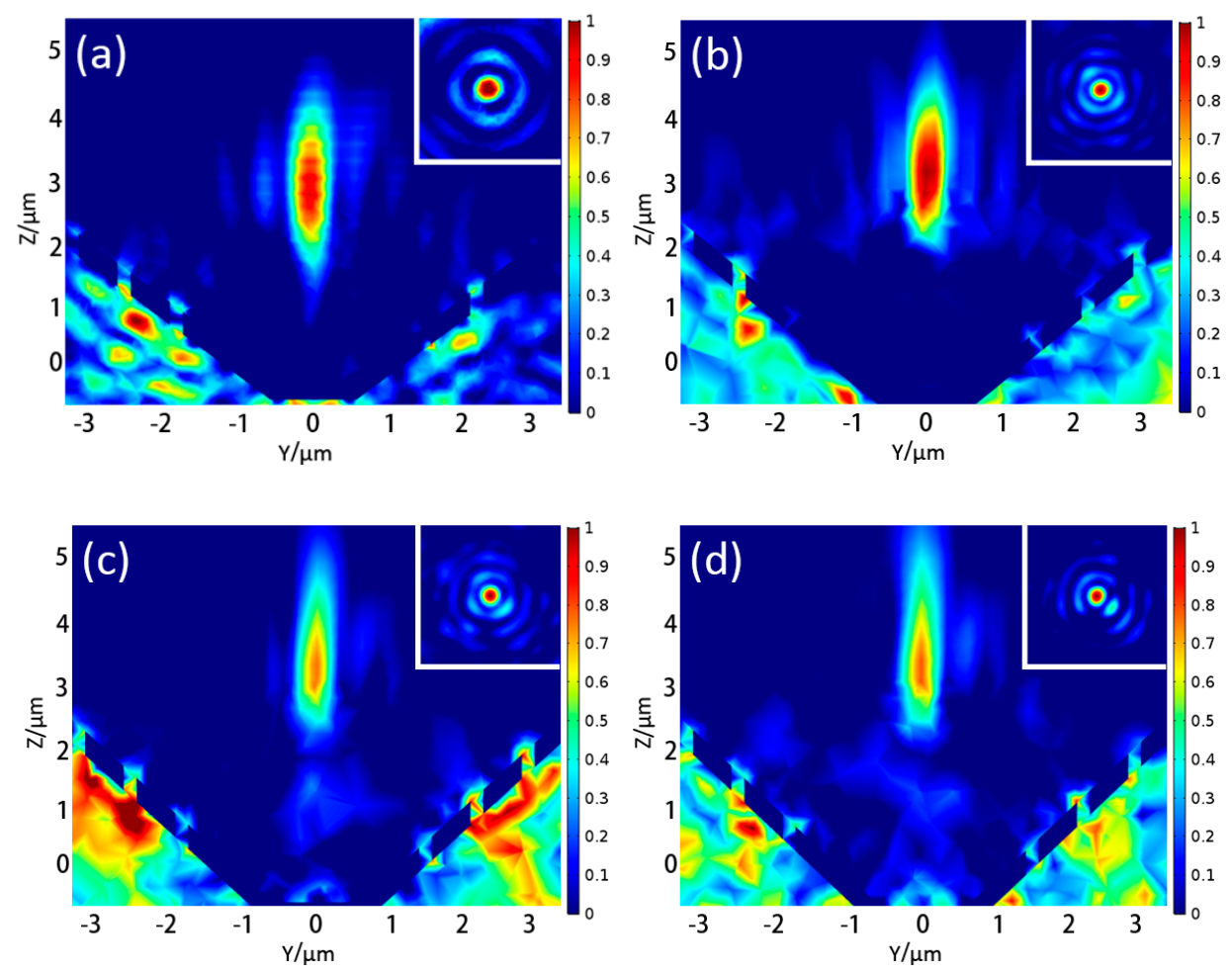

Figure 2. Normalized E-field at the $Y$-Z plane under excitation by left-hand circularly polarized light with gap $=800 \mathrm{~nm}$ for (a) $h_{0}=575 \mathrm{~nm}$, (b) $h_{0}=625 \mathrm{~nm}$, (c) $h_{0}=675 \mathrm{~nm}$, and (d) $h_{0}=725 \mathrm{~nm}$. The insets on the upper right represent the corresponding intensity distributions from the top view.

When the type of incident light is switched to plane waves with right-hand circular polarization, a plasmonic vortex field is generated around the origin of the structure that fits the second Bessel function of the first kind, as shown in Figure 4. In addition, when $h_{0}$ changes, the position of the plasmonic vortex varies with a similar trend to that of the focus under excitation by light with left-hand circular polarization. This longitudinal parameter $h_{0}$ thus makes the 3D PASL more flexible and the focal point or plasmonic vortex can therefore be adjusted in the longitudinal direction. As a result, 
the proposed 3D PASL is capable of creating a multi-functional application that will represent an aggressive expansion from the capabilities of the 2D PASL.
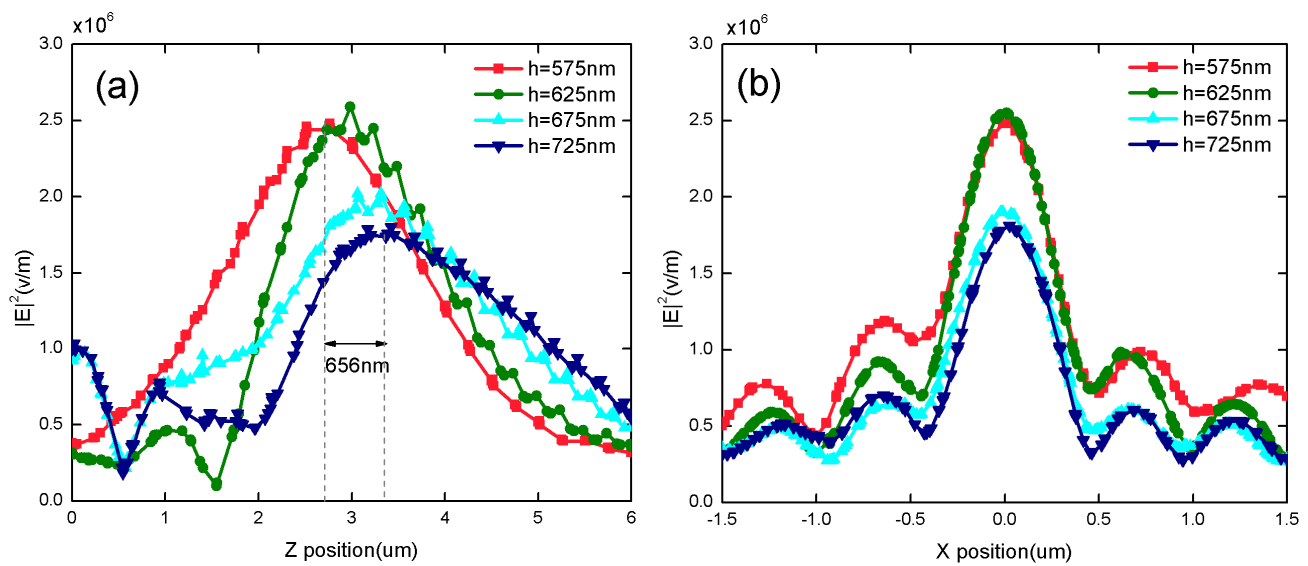

Figure 3. Focusing properties of 3D PASL with left-hand circularly polarized light excitation. The intensity profiles are shown (a) along the positive $z$-axis and (b) on the focal plane of the structure with gradually increasing values of $h_{0}$.
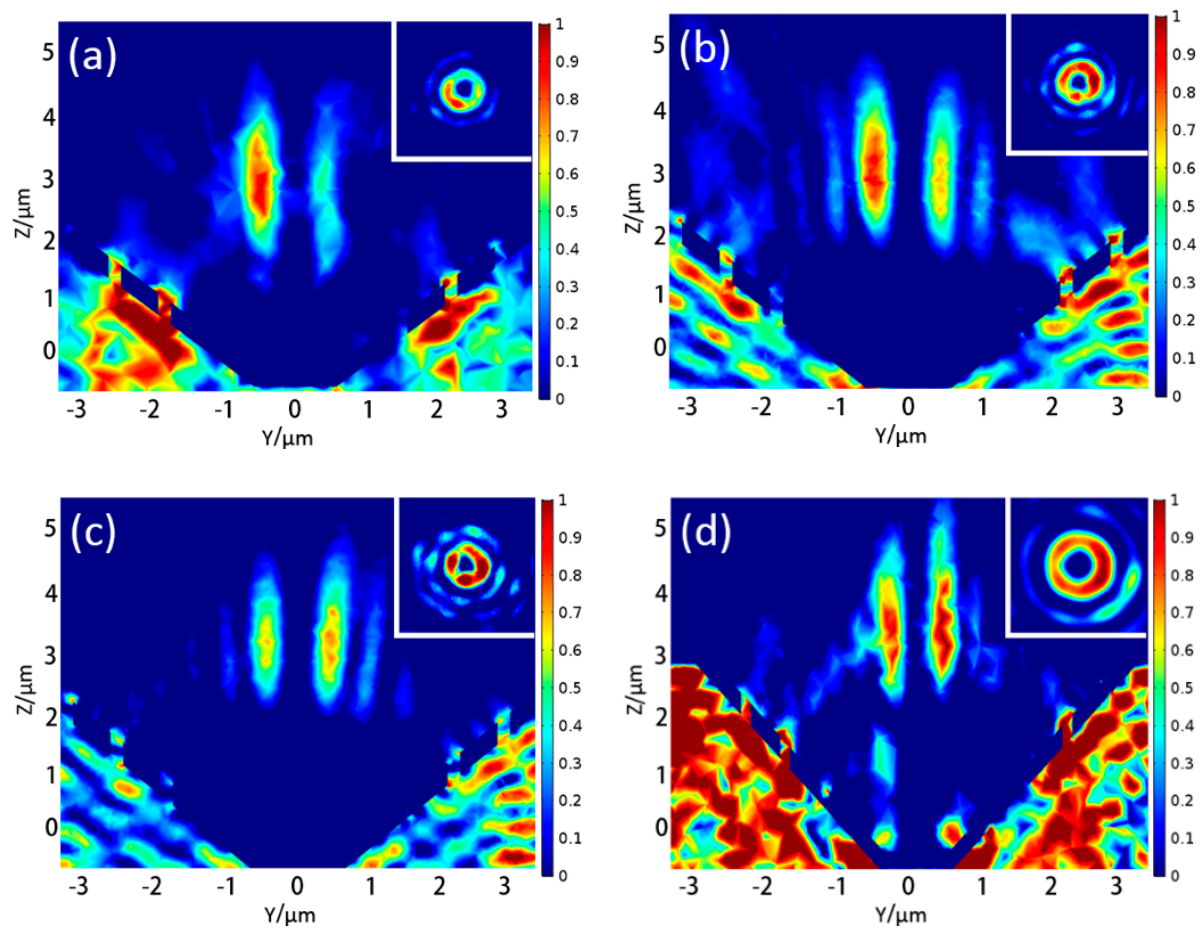

Figure 4. Normalized E-field at the $Y$ - $Z$ plane under right-hand circularly polarized light excitation with gap $=800 \mathrm{~nm}$ when (a) $h_{0}=575 \mathrm{~nm},(\mathbf{b}) h_{0}=625 \mathrm{~nm},(\mathbf{c}) h_{0}=675 \mathrm{~nm}$, and (d) $h_{0}=725 \mathrm{~nm}$.

We also investigate the feasibility of trapping and rotation of microparticles using the 3D PASL, as compared with the feasibility of using the previous 2D PASL. It is assumed that the microparticle to be used is a polystyrene sphere with a diameter of $1 \mu \mathrm{m}$ that is lying on the focal plane. Because of the three-dimensional structure of the PASL, we also analyzed the optical force acting in the $z$-direction, which helps to overcome the problem of particle manipulation on the surface when using the 2D PASL. The Maxwell stress tensor $\hat{T}_{i j}$ is used to calculate the optical force (F) and the optical trapping potential based on Equations (2)-(4) below. An integral stress tensor is applied over the entire spherical surface to obtain the optical force acting on the microsphere. The optical trapping potential is given by integrating the optical force acting on the microsphere along a specific direction. 


$$
\begin{gathered}
\hat{T}_{i j}=\left[\varepsilon E_{i} E_{j}+\mu_{0} H_{i} H_{j}-\frac{1}{2}\left(\varepsilon E^{2}+\mu_{0} H^{2}\right) \delta_{i j}\right] \\
F=\oint_{S} n \hat{T}_{i j} d S \\
U=\int F d l
\end{gathered}
$$

Here, $n$ is the unit vector in the normal direction of the closed surface that surrounds the target space. When the sphere moves on the $x$-axis in the focal plane, the optical forces that are exerted on the microsphere in the $x$-direction $(F x)$ and the $y$-direction $(F y)$ are as shown in Figure 5a. Obviously, $F x$ is much stronger than $F y$, and $F x$ plays a major role in pulling the microsphere back towards the center of the focus. When the sphere moves on the $y$-axis in the focal plane, Figure $5 c$ indicates that the optical force exerted on the microsphere in the $y$-direction $(F y)$ plays a major role and also pulls the microsphere back towards the center of the focus. In the vertical direction, as shown in Figure 5e, when the microsphere is below the focal point, the optical force that is exerted on the microsphere is positive along the $z$-axis, while the optical force that is exerted on the microsphere is negative along the $z$-axis when the microsphere is located above the focal point; this also means that the stable position of such a $1-\mu \mathrm{m}$-diameter microsphere is the focus. These results are consistent with the principle of optical tweezers, in either the lateral focal plane or the vertical focal plane. The calculated trapping potentials in the $x$-direction and the $y$-direction are shown in Figure $5 \mathrm{~b}, \mathrm{~d}$, respectively. Normally, we consider $10 k_{B} T$ as the threshold for stable optical trapping [15], where $k_{B}$ is the Boltzmann constant and $T$ is the thermodynamic temperature. We can show here that the potential well is about $250 k_{B} T$ in depth, which means that the formed plasmonic focus can capture a polystyrene sphere with a diameter of $1 \mu \mathrm{m}$ stably when the power of the incident light is $80 \mathrm{mw}$. So conventional lasers can generate sufficient optical force for stable optical trapping.

Next, we change the incident light to a right-hand circularly polarized plane wave and calculate the resulting optical force and potential. When a 1- $\mu \mathrm{m}$-diameter microsphere leaves the origin to travel from $-1.5 \mu \mathrm{m}$ to $1.5 \mu \mathrm{m}$ along the $y$-axis $/ x$-axis, the optical forces that are exerted on the microsphere are as shown in Figure 6a-c. We found that when the microsphere is located outside the plasmonic "doughnut", the optical forces will drag the microsphere into the ring. Note here that because of the asymmetry of the AS, the plasmonic vortex field is not a completely uniform ring. Therefore, the curves of Fx and Fy are not symmetrical with $x=0$ or $y=0$. In addition, as shown in Figure $6 \mathrm{~b}, \mathrm{~d}$, when the plasmonic vortex is generated by excitation with right-hand circularly polarized light, the depth of optical potential is greater than $30 k_{B} T$, i.e., the plasmonic vortex can trap the nanoparticle within the hollow of the doughnut shape.

Normally, spherical microparticles are commonly used as the objects to be captured and manipulated in optical trapping experiments. In addition, E. coli bacteria, cubic crystals, and ring particles can also be manipulated. Another very interesting nonspherical shape for use in these experiments is a cylinder, such as micro-nano metal rods or carbon nanotubes. These nonspherical particles provide more dynamic information than spherical particles, particularly in the case of optical vortex trapping.

Therefore, we also studied the optical torque acting when a cylindrical micro-rod is lying at the center of the 3D PASL at a rotation angle $\theta$. As is shown in Figure 7, the cylindrical micro-rod is in the $x-z$ plane and rotated around the $y$-axis. Here, the rotation angle $\theta$ is defined as the angle between the main axis of the cylinder and the $z$-axis, i.e., the angle between the dotted line and the $z$-axis in Figure 7 . The parameters of the 3D-PASL in this case are $h_{0}=625 \mathrm{~nm}$ and $g a p=800 \mathrm{~nm}$. The cross-sectional radius of the cylinder is $200 \mathrm{~nm}$, and its height is $1400 \mathrm{~nm}$. The cylinder is assumed lying at the center of the focus (under left-hand circularly polarized excitation) or the plasmonic vortex (under right-hand circularly polarized excitation). The optical torque $T$ that is exerted on the cylinder has also been 
calculated by integrating the Maxwell stress tensor $\hat{T}$ over a surface that encloses the object under study, as follows:

$$
T=-\oint_{S} n(\hat{T} \times r) d S
$$
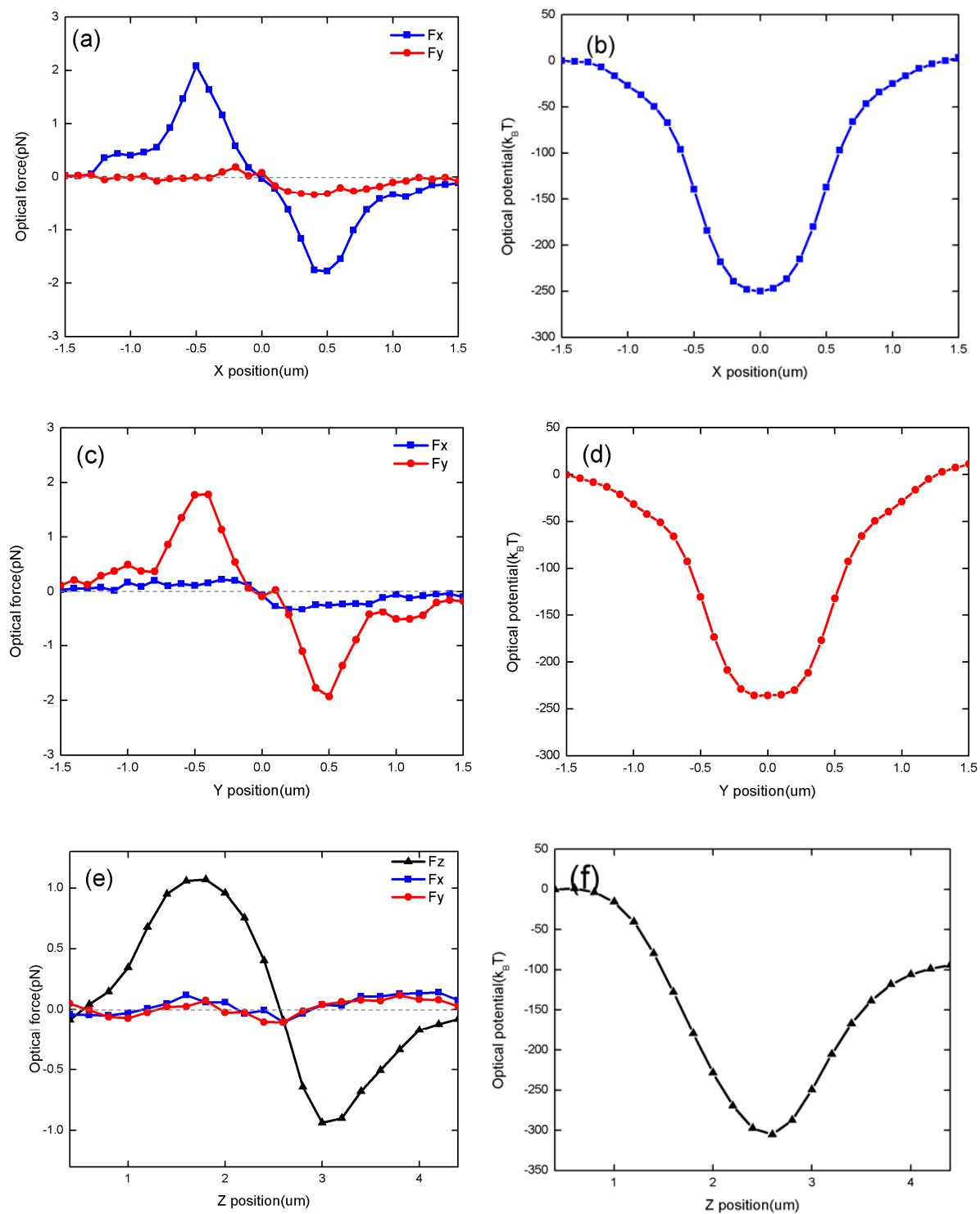

Figure 5. Optical trapping force exerted on a microsphere under left-hand circularly polarized light excitation. Please note that the focus is defined as the origin here. (a) Calculated optical trapping force in the $x$ and $y$ directions when the microsphere moves on the $x$-axis. (b) Calculated trapping potential when the microsphere moves on the $x$-axis. (c) Calculated optical trapping force in the $x$ and $y$ directions when the microsphere moves on the $y$-axis. (d) Calculated trapping potential when the microsphere moves on the $y$-axis. (e) Calculated optical forces in the $x, y$ and $z$ directions when the microsphere moves on the $z$-axis. (f) Calculated trapping potential in the $z$-direction. 

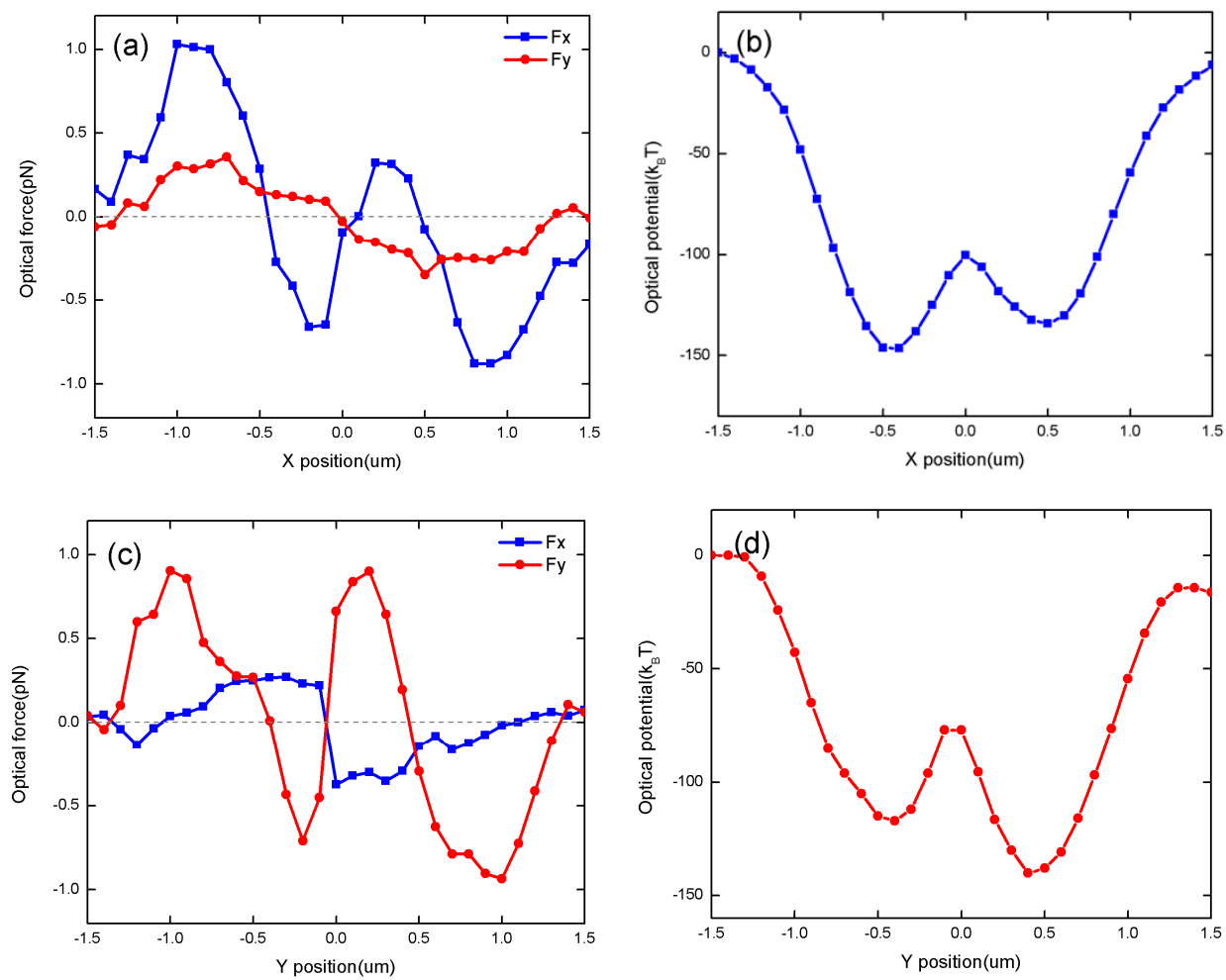

Figure 6. Optical trapping force exerted on the microsphere under right-hand circularly polarized excitation light. (a) Calculated optical trapping forces in the $x$ and $y$ directions when the microsphere moves on the $x$-axis. (b) Calculated trapping potential in the $x$-direction. (c) Calculated optical trapping forces in the $x$ - and $y$-directions when the microsphere moves on the $y$-axis. (d) Calculated trapping potential in the $y$-direction.

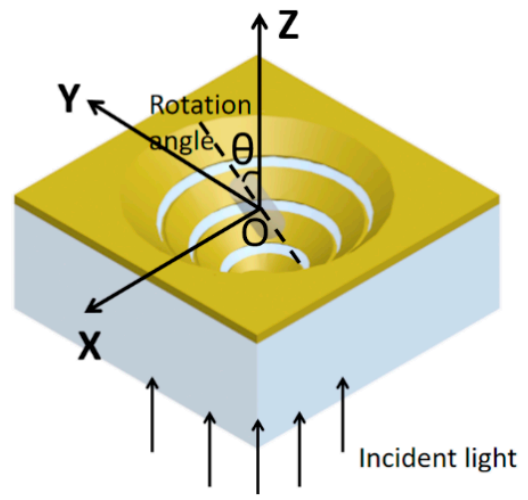

Figure 7. Schematic diagram of the rotational orientation of a cylindrical rod in the 3D PASL.

Here, $r$ is the moment arm in the angular momentum flux. The relationship between the optical torque and the rotation angle is shown in Figure $8 \mathrm{a}, \mathrm{b}$ under left-hand circularly polarized excitation and right-hand circularly polarized excitation conditions, respectively. Figure $8 \mathrm{a}$ shows that the torque in the $y$-direction plays a major role when the cylinder rotates around the $y$-axis. When the rotation angle ranges from 0 to $90^{\circ}, T y$ is greater than 0 , which means that the optical force tends to rotate the micro-rod towards the vertical direction (i.e., $\theta=0$ ). Additionally, when the rotation angle is between 0 and $-90^{\circ}$ (i.e., it is rotating in the opposite direction), $T y$ is less than 0 , which also indicates that the optical force tends to rotate the cylinder back towards the vertical direction. Therefore, when subjected to left-hand circularly polarized excitation, the optical force stabilizes the cylinder in the vertical direction at a rotation angle of $0^{\circ}$ with respect to the optical axis. When the cylinder was placed in the plasmonic vortex (i.e., under right-hand circularly polarized excitation), with results as shown in 
Figure $8 b$, we found that the optical torque in the $y$-direction reached a maximum when the rotation angle was approximately $\pm 45^{\circ}$, and the optical force then tends to rotate the rod towards the horizontal direction. However, the rotation angle at the maximum torque is different under the left-hand circularly polarized excitation conditions, this is because of the different beam waists of the focal point and the plasmonic vortex.
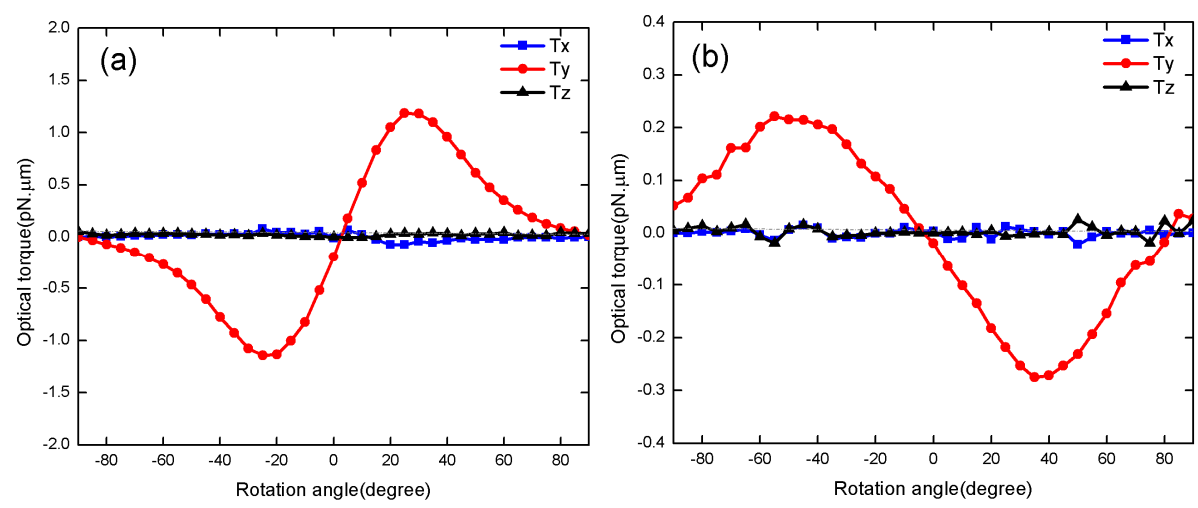

Figure 8. Optical torques of micro-cylinder at different rotation angles under (a) left-hand and (b) right-hand circularly polarized excitation.

\section{Conclusions}

We have investigated the optical performance of a three-turn 3D plasmonic Archimedes spiral lens under circularly polarized light illumination by numerical simulations without conducting experiments. This type of miniature plasmonic structure can either generate a focal point at the center of the structure under excitation by a left-hand circular polarization plane wave or a plasmonic vortex under excitation by a right-hand circular polarization plane wave. Therefore, this structure may have many more new potential applications than the traditional 2D planar plasmonic Archimedes spiral lens. By adjusting the parameters of the structure, such as the rise height $h_{0}$ of the AS, or by switching the circular polarization, the 3D PASL can be reconfigured flexibly for use in practical applications. We have analyzed the important factors for control of the SPP field in detail, and found that the focus and the vortex length both increase with increments in $h_{0}$. Finally, we investigated the stability of the device for trapping and rotation of microparticles suspended in PBS buffer solution. Additionally, the optical torque (T) of a cylindrical micro-rod when tilted at different rotation angles is discussed for rotation applications; the results provide a theoretical basis for potential applications of this structure in biomedicine and microbiology in the future. This 3D PASL structure may find further applications in plasmonic-assisted optical trapping and enhanced spectroscopy techniques.

Author Contributions: Conceptualization, L.C. and X.L.; Methodology, L.C. and X.L.; Formal Analysis, L.C., X.L. and Z.W.; Writing-Original Draft Preparation, X.L.; Writing-Review \& Editing, L.C., X.L., Z.W., P.C., X.H. and T.N.

Funding: This work was founded by the Natural Science Foundation of Gansu Province, grant number 1606RJZA068; the National Natural Science Foundation of China, grant number 61701207, and the Fundamental Research Funds for the Central Universities, grant number 2016-143.

Conflicts of Interest: The authors declare no conflict of interest.

\section{References}

1. Sambles, J.R.; Bradbery, G.W.; Yang, F. Optical excitation of surface plasmons: An introduction. Contemp. Phys. 1991, 32, 173-183. [CrossRef]

2. Maier, S.A. Plasmonics: Fundamentals and Applications; Springer: Berlin, Germany, 2007; Volume 52, pp. 49-74.

3. Hecht, B.; Bielefeldt, H.; Novotny, L.; Inouye, Y.; Pohl, D.W. Local Excitation, Scattering, and Interference of Surface Plasmons. Phys. Rev. Lett. 1996, 77, 1889-1892. [CrossRef] [PubMed] 
4. Kawata, S.; Inouye, Y.; Verma, P. Plasmonics for near-field nano-imaging and superlensing. Nat. Photonics 2009, 3, 388. [CrossRef]

5. Barnes, W.L.; Dereux, A.; Ebbesen, T.W. Surface plasmon subwavelength optics. Nature 2003, 424, 824-830. [CrossRef] [PubMed]

6. Antosiewicz, T.J.; Wróbel, P.; Szoplik, T. Performance of Scanning Near-Field Optical Microscope Probes with Single Groove and Various Metal Coatings. Plasmonics 2011, 6, 11-18. [CrossRef] [PubMed]

7. Cheng, L.; Cao, P.; Li, Y.; Kong, W.; Zhao, X.; Zhang, X. High Efficient Far-Field Nanofocusing with Tunable Focus Under Radial Polarization Illumination. Plasmonics 2012, 7, 175-184. [CrossRef]

8. Wei, W.; Guo, Z.; Li, R.; Zhang, J.; Yi, L.; Wang, X.; Qu, S. Ultra-thin, planar, broadband, dual-polarity plasmonic metalens. Photonics Res. 2015, 3, 68-71.

9. Ritchie, R.H.; Arakawa, E.T.; Cowan, J.J.; Hamm, R.N. Surface-Plasmon Resonance Effect in Grating Diffraction. Phys. Rev. Lett. 1968, 21, 1530-1533. [CrossRef]

10. Li, J.; Yang, C.; Li, J.; Li, Z.; Zu, S.; Song, S.; Zhao, H.; Lin, F.; Zhu, X. Plasmonic Focusing in Nanostructures. Plasmonics 2014, 9, 879-886. [CrossRef]

11. Kim, H.; Park, J.; Cho, S.W.; Lee, S.Y.; Kang, M.; Lee, B. Synthesis and dynamic switching of surface plasmon vortices with plasmonic vortex lens. Nano Lett. 2010, 10, 529-536. [CrossRef]

12. Homola, J.; Yee, S.S.; Gauglitz, G. Surface plasmon resonance sensors: Review. Anal. Bioanal. Chem. 1999, 377, 528-539. [CrossRef] [PubMed]

13. Kwak, E.S.; Onuta, T.D.; Amarie, D.; Potyrailo, R.; Stein, B.; Jacobson, S.C.; Schaich, W.L.; Dragnea, B. Optical Trapping with Integrated Near-Field Apertures. J. Phys. Chem. B 2004, 108, 13607. [CrossRef]

14. Righini, M.; Zelenina, A.S.; Girard, C.; Quidant, R. Parallel and selective trapping in a patterned plasmonic landscape. Nat. Phys. 2007, 3, 477-480. [CrossRef]

15. Ashkin, A.; Dziedzic, J.M.; Bjorkholm, J.E.; Chu, S. Observation of a single-beam gradient force optical trap for dielectric particles. Opt. Lett. 1986, 11, 288. [CrossRef] [PubMed]

16. Chen, J.; Wang, C.; Lu, G.; Li, W.; Xiao, J.; Gong, Q. Highly efficient nanofocusing based on a T-shape micro-slit surrounded with multi-slits. Opt. Express 2012, 20, 17734-17740. [CrossRef]

17. Hu, C.; Ji, X.; Ding, J. Focusing properties of a novel plasmonic lens allowing for circularly polarized illumination. J. Mod. Opt. 2017, 64, 1604-1613. [CrossRef]

18. Ditlbacher, H.; Krenn, J.R.; Schider, G.; Leitner, A.; Aussenegg, F.R. Two-Dimensional Optics with Surface Plasmon Polaritons. Appl. Phys. Lett. 2002, 81, 1762-1764. [CrossRef]

19. Juan, M.L.; Gordon, R.; Pang, Y.; Eftekhari, F.; Quidant, R. Self-induced back-action optical trapping of dielectric nanoparticles. Nat. Phys. 2009, 5, 915. [CrossRef]

20. Tomoki, O.; Shintaro, M. Study of surface plasmon chirality induced by Archimedes' spiral grooves. Opt. Express 2006, 14, 6285-6290.

21. Ku, C.T.; Lin, H.N.; Huang, C.B. Direct observation of surface plasmon vortex and subwavelength focusing with arbitrarily-tailored intensity patterns. Appl. Phys. Lett. 2015, 106, 053112. [CrossRef]

22. Cho, S.W.; Park, J.; Lee, S.Y.; Kim, H.; Lee, B. Coupling of spin and angular momentum of light in plasmonic vortex. Opt. Express 2012, 20, 10083. [CrossRef] [PubMed]

23. Chen, C.F.; Ku, C.T.; Tai, Y.H.; Wei, P.K.; Lin, H.N.; Huang, C.B. Creating optical near-field orbital angular momentum in a gold metasurface. Nano Lett. 2015, 15, 2746-2750. [CrossRef] [PubMed]

24. Tsai, W.Y.; Huang, J.S.; Huang, C.B. Selective trapping or rotation of isotropic dielectric microparticles by optical near field in a plasmonic archimedes spiral. Nano Lett. 2014, 14, 547. [CrossRef] [PubMed]

25. Spektor, G.; Kilbane, D.; Mahro, A.K.; Frank, B.; Ristok, S.; Gal, L.; Kahl, P.; Podbiel, D.; Mathias, S.; Giessen, H. Revealing the subfemtosecond dynamics of orbital angular momentum in nanoplasmonic vortices. Science 2017, 355, 1187-1191. [CrossRef] [PubMed]

26. Hiroo, U.; Hiroki, K. Optical rotor capable of controlling clockwise and counterclockwise rotation in optical tweezers by displacing the trapping position. Appl. Opt. 2010, 49, 1991-1996.

27. Wang, M.D.; Yin, H.; Landick, R.; Gelles, J.; Block, S.M. Stretching DNA with optical tweezers. Biophys. J. 1997, 72, 1335-1346. [CrossRef]

28. Gore, J.; Bryant, Z.; Nöllmann, M.; Le, M.U.; Cozzarelli, N.R.; Bustamante, C. DNA overwinds when stretched. Nature 2006, 442, 836. [CrossRef]

29. Schultz, D.A. Plasmon resonant particles for biological detection. Curr. Opin. Biotechnol. 2003, 14, 13-22. [CrossRef] 
30. Yang, S.; Chen, W.; Nelson, R.L.; Zhan, Q. Miniature circular polarization analyzer with spiral plasmonic lens. Opt. Lett. 2009, 34, 3047-3049. [CrossRef]

31. Palik, E.D. Handbook of Optical Constants of Solids II; Boston Academic Press: Boston, MA, USA, 1991; Volume 1, pp. 77-135. 